\title{
Cardiology
}

\section{A Risk Prediction Model for Mortality in Patients with Dilated Cardiomyopathy}

\author{
Nuraly A. Kurbanov, PhD; Ravshanbek D. Kurbanov, PhD, ScD; \\ Timur A. Abdullaev, PhD, ScD; Igor A. Tsoy, PhD*; Regina Sh. Bekbulatova, PhD \\ Republican Specialized Center of Cardiology \\ Tashkent, Uzbekistan
}

\begin{abstract}
The aim of this study was to build a risk prediction model for 1-year mortality on the bases of clinical and functional indicators in patients with dilated cardiomyopathy (DCM).

Materials and Methods: We examined 205 patients (aged from 16 to 61 years) with DCM and chronic heart failure in NYHA FC II-IV. The study included the collection of anamnestic data, physical examinations, clinical and biochemical laboratory methods, 12-lead ECG, echocardiography, Holter ECG monitoring, and 6-minute walk test. The duration of follow-up was 3 to 170 months (36.7 \pm 5.6 ). The study end-points were death (sudden death or progression of heart failure) and thromboembolic complications (pulmonary embolism, ischemic stroke, thromboembolism of the vessels of kidneys and lower extremities).

Results: A sequential evaluation of clinical-anamnestic and instrumental data with analysis of the life expectancy and the subsequent construction of a risk prediction model for 1-year mortality by the method of multiple stepwise logistic regression was performed. In accordance with the results of multiple regression analysis, among the clinico-functional parameters, NYHA class III/IV, low blood pressure, a relatively young age, abnormal QRS complex, high-grade ventricular arrhythmias and an increase in LVESV/LVEDV ratio ( $>0.66)$ are the most influencing factors for a fatal outcome within 1 year of observation. (International Journal of Biomedicine. 2018;8(2):118-122.)
\end{abstract}

Key Words: dilated cardiomyopathy $\bullet$ risk stratification $\bullet$ life expectancy $\bullet$ prognosis

\section{Abbreviations}

BP, blood pressure; CHF, chronic heart failure; DCM, dilated cardiomyopathy; FC, functional class; HF, heart failure; LV, left ventricle; LVEF, leftventricularejection fraction;LVESV, leftventricularend-systolic volume; LVEDV, left ventricularend-diastolic volume; MRA, multiple regression analysis; PVC, premature ventricular contraction; RF, risk factor; VA, ventricular arrhythmia.

\section{Introduction}

The predominant cause of chronic heart failure (CHF) with reduced LVEF is ischemic heart disease $(70 \%$ of all cases) and dilated cardiomyopathy (DCM) (13\%-15\% of all cases) ${ }^{(1,2)}$ Previous epidemiological studies have shown that the prognosis of life for patients with CHF of ischemic etiology is significantly worse compared to patients with non-

*Corresponding author: Igor A. Tsoy, PhD. Republican Specialized Center of Cardiology, Tashkent, Uzbekistan. E-mail: tsoigor@inbox.ru ischemic heart failure (HF). ${ }^{(3)} \mathrm{A}$ widespread implementation of standards for the management of CHF and surgical methods (aorta-coronary bypass, coronary angioplasty) has significantly improved the prognosis and life expectancy of patients with non-ischemic CHF. ${ }^{(4)}$ In the treatment of $\mathrm{CHF}$ associated with DCM, in addition to resynchronizing therapy, operations to reverse remodeling and heart transplantation contribute to the improvement of the quality of life and life expectancy. ${ }^{(5,6)}$ However, radical methods for DCM treatment have not been developed to date. In this regard, the search for predictors of an unfavorable course of the disease and the identification of patients at high risk of death remains an important task. 
The aim of this study was to build a risk prediction model for 1-year mortality on the bases of clinical and functional indicators in DCM patients.

\section{Materials and Methods}

Between 1998 and 2012, 205 patients (aged from 16 to 61 years) with DCM and CHF in NYHA FC II-IV were examined. DCM was diagnosed in accordance with the WHO criteria (1995). ${ }^{(7)}$ The study included the collection of anamnestic data, physical examinations, clinical and biochemical laboratory methods, 12-lead ECG, echocardiography, Holter ECG monitoring (HEM), and 6-minute walk test (6MWT); ${ }^{(8)}$ if necessary, coronary angiography was performed to exclude coronary artery disease. The duration of follow-up was 3 to 170 months (36.7 \pm 5.6$)$. The study end-points were death (sudden death or progression of $\mathrm{HF}$ ) and thromboembolic complications (pulmonary embolism, ischemic stroke, thromboembolism of the vessels of kidneys and lower extremities).

The study was approved by the Ethics Committee of Republican Specialized Center of Cardiology (Uzbekistan). Written informed consent was obtained from each patient.

Statistical analysis was performed using statistical software package SPSS version 20.0 (SPSS Inc, Chicago, IL). Baseline characteristics were summarized as frequencies and percentages for categorical variables and as mean $\pm \mathrm{SD}$ for continuous variables. Odds ratios (ORs) and their 95\% confidence intervals (CIs) were calculated by using logistic regression. Categorical variables were analyzed using the Chi-square test. A probability value of $P<0.05$ was considered statistically significant.

\section{Results}

To solve the research goal, we performed a 5-year prospective observation. By the end of the first year of observation, 55(26.8\%) patients had died; by the end of 2 years - 34 more (22.7\% of the remaining 150 patients). The total mortality for the next 3-5 years of follow-up was $44.8 \%$ (52 of 116 patients). By the end of the 5-year observation, less than one-third of patients remained alive $(64 / 31.2 \%$ of 205 patients). The main causes of death were the progression of HF and sudden death. Death from thromboembolic complications was $2 \%-9 \%$.

In order to identify significant predictors of mortality in DCM patients, 76 indices (anamnestic data, parameters of clinical examination, ECG, echocardiography, HEM, questionnaires, functional tests, and immunological markers) obtained during a primary examination were analyzed. The value of each indicator was presented as a dichotomous sign by means of coding. For qualitative indicators, the encoding was as follows: "1" - a sign (+); "0" - a sign (-).

In cases of continuous quantitative variables, to obtain threshold values, we performed tabulation with a division of the entire range into intervals followed by their conversion: " 1 " is a conditionally pathological value; " 0 " is a conditionally non-pathological value.

In the same way, study end-points were encoded: "1" - event (death) occurred; "0" - event (death) did not occur. The results of encoding the qualitative and quantitative variables are presented in Table 1 .

Table 1.

Encoding of clinical and functional indicators represented by continuous variables

\begin{tabular}{|l|c|c|}
\hline \multicolumn{1}{|c|}{ Variable } & $\begin{array}{c}\text { Code } « 1 » \\
\text { a sign }(+)\end{array}$ & $\begin{array}{c}\text { Code } « 0 » \\
\text { a sign }(-)\end{array}$ \\
\hline Age & $<40$ years & $>40$ years \\
\hline FC of HF (NYHA) & $\geq$ III & $<$ III \\
\hline DCM variant & family variant & other variants \\
\hline $\begin{array}{l}\text { Blockade of the left/right leg of the } \\
\text { bundle of His and/or the presence of } \\
\text { pseudo-infarct Q wave/QS in ECG }\end{array}$ & + & - \\
\hline PVCs II-IV A-B grades & + & - \\
\hline LVEF, \% & $<34 \%$ & $>34 \%$ \\
\hline LVESV, ml & $>178 \mathrm{ml}$ & $<178 \mathrm{ml}$ \\
\hline LVESV/LVEDV & $>0.66$ & $<0.66$ \\
\hline $\begin{array}{l}\text { Fatal outcome within 1 year of } \\
\text { observation }\end{array}$ & + & - \\
\hline
\end{tabular}

At the initial stage of this part of the study, the relationship between each of the studied clinico-functional indicators and death within 1 year of observation was assessed using the Pearson $\chi^{2}$ criterion and univariate regression analysis. The odds ratio (OR) of death was evaluated as the exponential coefficient $(\operatorname{Exp}(\beta))$ of the regression equation. Nine of 76 indicators had OR $>1$. In all these cases, 95\% confidence intervals of ORs did not cross the value $<1$. Therefore, each of these 9 indicators was considered as RF for death (Table 2).

Table 2.

Risk factors for death within 1 year of observation. Results of univariate regression analysis

\begin{tabular}{|l|l|c|c|c|c|}
\hline $\mathrm{N}$ & \multicolumn{1}{|c|}{ RF for death } & $\chi 2$ & $\begin{array}{c}\text { OR } \\
(\operatorname{Exp}(\beta))\end{array}$ & $95 \% \mathrm{CI}$ & $P$-value \\
\hline 1 & Age $<40$ years & $15.4(P<0.01)$ & 6.1 & $2.9-37.1$ & $<0.05$ \\
\hline 2 & Family form of DCM & $18.2(P<0.01)$ & 10.1 & $3.1-42.4$ & $<0.05$ \\
\hline 3 & NYHA FC $\geq$ III & $10.8(P<0.01)$ & 6.3 & $2.8-16.2$ & $<0.05$ \\
\hline 4 & SBP $<100$ mmHg & $4.9(P<0.05)$ & 2.6 & $1.6-10.2$ & $<0.05$ \\
\hline & $\begin{array}{l}\text { Blockade of the left/ } \\
\text { right leg of the bundle } \\
\text { of His and/or the } \\
\text { presence of pseudo- } \\
\text { infarct Q wave in ECG }\end{array}$ & $3.8(P<0.05)$ & 1.8 & $1.2-6.4$ & $<0.05$ \\
\hline 6 & $\begin{array}{l}\text { High-grade VAs } \\
7\end{array}$ & $4.5(P<0.05)$ & 2.4 & $1.5-9.9$ & $<0.05$ \\
\hline 8 & LVESV $>178$ ml & $2.0(P<0.05)$ & 1.5 & $1.7-4.5$ & $<0.05$ \\
\hline 9 & $\begin{array}{l}\text { LVEF }<34 \% \\
>0.66\end{array}$ & $2.5(P<0.05)$ & 1.5 & $1.2-4.8$ & $<0.05$ \\
\hline
\end{tabular}

The results of regression analysis indicate that the risk of death increases 10 times for the familial DCM and 6 times 
for young patients and patients with high FC of HF. RFs such as low BP, pathological changes in the QRS complex in ECG, high-grade PVCs, low LVEF and increased LVESV increase the risk of death by 1.5-2.5 times.

The next stage of the study was the construction of a risk prediction model for 1-year mortality. For this, the stepwise logistic regression procedure of MRA was used. To create a predictive model, the logistic regression equation was used:

Odds $(y \neq 1)=e\left(\beta_{0}+\beta_{1} x_{1}+\ldots . . \beta_{k} x_{k}\right)$, where

Odds - chance;

$\beta_{0}$ - exponential coefficient of the regression equation (constant);

$\beta_{1}-\beta_{\mathrm{k}}-$ coefficients of the regression equation that require calculation;

$\mathrm{x}_{1}-\mathrm{x}_{\mathrm{k}}$ - the significance of risk factors;

The probability of fatal outcome $(\mathrm{P})$ was determined by the formula: $\mathrm{P}=$ odds/odds +1 .

The results of this section of the study are presented in Table 3.

Table 3.

Risk factors for death within 1 year of observation. Results of MRA

\begin{tabular}{|c|l|c|c|c|c|c|c|}
\hline $\mathrm{N}$ & \multicolumn{1}{|c|}{$\mathrm{RF}$} & & $\mathrm{K} \beta$ & $\beta$ exp & $95 \%$ CI & -value & Score \\
\hline 1 & NYHA FC $\geq \mathrm{III}$ & $\mathrm{x}_{1}$ & 2.58 & 14.65 & $4.6-48.5$ & $<0.01$ & 15 \\
\hline 2 & Age $<40$ years & $\mathrm{x}_{2}$ & 1.52 & 5.03 & $2.7-16.2$ & $<0.01$ & 5 \\
\hline 3 & SBP $<100 \mathrm{mmHg}$ & $\mathrm{x}_{3}$ & 1.44 & 4.26 & $1.6-11.8$ & $<0.01$ & 4 \\
\hline $\begin{array}{l}\text { Blockade of the left/ } \\
\text { right leg of the bundle of } \\
4\end{array}$ & $\begin{array}{l}\text { His and/or the presence } \\
\text { of pseudo-infarct Q } \\
\text { wave in ECG }\end{array}$ & $\mathrm{x}_{4}$ & 1.42 & 3.95 & $1.5-10.2$ & $<0.01$ & 4 \\
\hline 5 & High-grade VAs & $\mathrm{x}_{5}$ & 1.58 & 5.29 & $3.4-18.9$ & $<0.01$ & 5 \\
\hline 6 & LVESV/LVEDV $>0.66$ & $\mathrm{x}_{6}$ & 1.18 & 3.54 & $1.4-9.8$ & $<0.01$ & 4 \\
\hline
\end{tabular}

Thus, based on the results of MRA, it was noted that the most significant contributors to the probability of death in one year in DCM patients were NYHA classes III-IV, young age, low BP, the blockade of the left/right leg of the bundle of His and/or the presence of pseudo-infarct Q wave/QS in ECG, high-grade PVCs and LVESV/LVEDV ratio $>0.66$.

Using the obtained coefficients of the multifactorial logistic regression equation, the probability of a fatal outcome within the next year was calculated for all possible combinations of RFs (Table 4). As can be seen from Table 1, the probability of fatal outcome (P) was $0.015-0.023$ with one RF up to 0.941 with a full set of prognostic signs. In this connection, the entire range of $\mathrm{P}$ values (death risk) was divided into conditional intervals: $<0.21$ (minimal risk), 0.21-0.40 (average risk), 0.41-0.70 (high risk) and $>0.70$ (very high risk).

To simplify the calculation of OR for death in one year, a score-scale was introduced reflecting the conditional contribution of each indicator to the likelihood of death. The number of scores for each RF corresponded to the value of $\operatorname{Exp}(\beta)$ «restored» to an integer. Then the sums of the conditional scores and $\mathrm{P}$ values were compared. Thus, the differentiation of the risk degree, expressed in scores, had the following form: $\leq 15$ scores - a low risk of death; 16-22 scores - an average risk of death; 23-28 scores - a high risk of death; $>28$ scores - a very high risk of death. The assessment of the risk of death for the coming year, based on the summation of conventional scores, is simple and convenient for use in clinical practice (Table 5).

\section{Discussion}

Identification of factors contributing to a decrease in life expectancy or increased hospitalization in patients with CHF, allows us to adjust the strategy of treatment. ${ }^{(5)}$ Identification of patients with a low probability of survival helps clinicians to solve the problem of certain therapeutic interventions and to choose palliative management in case of an unfavorable shortterm prognosis. Conversely, patients with more optimistic prognostic indicators may be candidates for more "aggressive" therapeutic tactics, such as implantation of a cardioverterdefibrillator, artificial LV, etc. ${ }^{(9)}$

Thus, based on the results of MRA, it was noted that the most significant contributors to the probability of death in one year in DCM patients were NYHA classes III-IV, young age, low BP, the blockade of the left/right leg of the bundle of His and/or the presence of pseudo-infarct Q wave/QS in ECG, highgrade PVCs and LVESV/LVEDV ratio $>0.66$. At the same time, the frequency of fatal outcomes increased proportionally with the increase in the number of signs revealed during the primary examination. Analysis of all unfavorable indicators directly affecting the prognosis of life in DCM patients, especially the presence of two or more prognostic adverse factors in the patient, gives more grounds for a worse prognosis.

Our data largely coincide with the research data by Janashia and colleagues, ${ }^{(10)}$ which showed that a more serious prognosis in DCM patients was associated with the presence of a blockade of the left leg of the bundle of His, paroxysms of ventricular tachycardia and NYHA FC-IV. Our results showed that the majority of young people (under the age of 40) who died did so during the first 2 years of observation, which is statistically significant, while in the older age group, life expectancy was twice as high, which indicates a rapid progression of the pathological process in young patients. Along with the above factors of unfavorable prognosis in patients with CHF in many epidemiological studies, hypotension is an important factor reducing the chance of survival in CHF patients..$^{(3)}$ In this case, the negative effect of hypotension on the prognosis of patients with a reduced LVEF does not depend on CHF etiology. The data of these studies confirm our results: in DCM patients with hypotension, the lethal outcome occurred more often and earlier than in patients with normal BP.

According to data of a number of researchers, the state of the contractile function of LV has the main role in determining the prognosis of life in DCM patients. ${ }^{(11,12)}$ The results of our study showed that a more significant prognostic indicator was an increase in the LVESV/LVEDV ratio $(>0.66)$ due to a relatively higher LVESV, indicating a worsening of LV emptying during the systole period. H.Wite and co-authors ${ }^{(13)}$ also convincingly showed that an increase in LVESV affects negatively the prognosis and subsequent survival. The preserved LVEF and a small LV volume significantly improve the prognosis in DCM patients. ${ }^{(14)}$ 
Table 4.

The probability of a fatal outcome within the next year for all possible combinations of RFs

\begin{tabular}{|c|c|c|c|c|c|c|c|c|c|c|c|c|c|c|c|c|c|c|c|c|c|c|c|c|c|c|}
\hline $\mathrm{N}$ & $\mathrm{x}_{1}$ & $\mathrm{x}_{2}$ & $\mathrm{x}_{3}$ & $\mathrm{x}_{4}$ & $\mathrm{x}_{5}$ & $\mathrm{x}_{6}$ & $\mathrm{P}$ & Score & $\mathrm{N}$ & $\mathrm{x}_{1}$ & $x_{2}$ & $\mathrm{x}_{3}$ & $\mathrm{x}_{4}$ & $\mathrm{x}_{5}$ & $\mathrm{x}_{6}$ & $\mathrm{P}$ & Score & $\mathrm{N}$ & $x_{1}$ & $x_{2}$ & $\mathrm{x}_{3}$ & $\mathrm{x}_{4}$ & $\mathrm{X}_{5}$ & $x_{6}$ & $\mathrm{P}$ & Score \\
\hline 1 & 1 & & & & & & 0.023 & 15 & 23 & 1 & 1 & & 1 & & & 0.377 & 24 & 45 & 1 & 1 & & 1 & 1 & & 0.645 & 29 \\
\hline 2 & & 1 & & & & & 0.012 & 5 & 24 & 1 & 1 & & & 1 & & 0.338 & 25 & 46 & 1 & 1 & & & 1 & 1 & 0.512 & 29 \\
\hline 3 & & & 1 & & & & 0.017 & 4 & 25 & 1 & 1 & & & & 1 & 0.259 & 24 & 47 & 1 & 1 & & 1 & & 1 & 0.554 & 28 \\
\hline 4 & & & & 1 & & & 0.016 & 4 & 26 & & 1 & 1 & 1 & & & 0.190 & 13 & 48 & & 1 & 1 & 1 & 1 & & 0.386 & 18 \\
\hline 5 & & & & & 1 & & 0.015 & 5 & 27 & & 1 & 1 & & 1 & & 0.170 & 14 & 49 & & 1 & 1 & 1 & & 1 & 0.359 & 17 \\
\hline 6 & & & & & & 1 & 0.015 & 4 & 28 & & 1 & 1 & & & 1 & 0.120 & 13 & 50 & & 1 & 1 & & 1 & 1 & 0.372 & 18 \\
\hline 7 & 1 & 1 & & & & & 0.145 & 20 & 29 & & & 1 & 1 & 1 & & 0.098 & 13 & 51 & & 1 & & 1 & 1 & 1 & 0.342 & 18 \\
\hline 8 & 1 & & 1 & & & & 0.092 & 19 & 30 & & & 1 & 1 & & 1 & 0.115 & 12 & 52 & 1 & & 1 & 1 & 1 & & 0.521 & 28 \\
\hline 9 & 1 & & & 1 & & & 0.077 & 19 & 31 & & & & 1 & 1 & 1 & 0.107 & 13 & 53 & 1 & & & 1 & 1 & 1 & 0.384 & 28 \\
\hline 10 & 1 & & & & 1 & & 0.066 & 20 & 32 & 1 & & 1 & 1 & & & 0.265 & 23 & 54 & 1 & & 1 & & 1 & 1 & 0.385 & 28 \\
\hline 11 & 1 & & & & & 1 & 0.058 & 19 & 33 & 1 & & 1 & & 1 & & 0.233 & 24 & 55 & 1 & & 1 & 1 & & 1 & 0.426 & 27 \\
\hline 12 & & 1 & 1 & & & & 0.25 & 9 & 34 & 1 & & 1 & & & 1 & 0.190 & 23 & 56 & & & 1 & 1 & 1 & 1 & 0.443 & 17 \\
\hline 13 & & 1 & & 1 & & & 0.042 & 9 & 35 & 1 & & & 1 & 1 & & 0.201 & 24 & 57 & 1 & & 1 & 1 & 1 & 1 & 0.692 & 32 \\
\hline 14 & & 1 & & & 1 & & 0.036 & 10 & 36 & 1 & & & 1 & & 1 & 0.167 & 23 & 58 & 1 & 1 & & 1 & 1 & 1 & 0.789 & 33 \\
\hline 15 & & 1 & & & & 1 & 0.025 & 9 & 37 & 1 & & & & 1 & 1 & 0.148 & 24 & 59 & 1 & 1 & 1 & & 1 & 1 & 0.818 & 33 \\
\hline 16 & & & 1 & 1 & & & 0.021 & 8 & 38 & & 1 & & & 1 & 1 & 0.110 & 14 & 60 & 1 & 1 & 1 & 1 & & 1 & 0.842 & 32 \\
\hline 17 & & & 1 & & 1 & & 0.015 & 9 & 39 & & 1 & & 1 & & 1 & 0.102 & 13 & 61 & & 1 & 1 & 1 & 1 & 1 & 0.789 & 22 \\
\hline 18 & & & 1 & & & 1 & 0.018 & 8 & 40 & & 1 & & 1 & 1 & & 0.134 & 14 & 62 & 1 & 1 & 1 & 1 & 1 & & 0.897 & 33 \\
\hline 19 & & & & 1 & 1 & & 0.018 & 9 & 41 & & & 1 & & 1 & 1 & 0.124 & 13 & 63 & 1 & 1 & 1 & 1 & 1 & 1 & 0.941 & 37 \\
\hline 20 & & & & 1 & & 1 & 0.017 & 8 & 42 & 1 & 1 & 1 & 1 & & & 0.721 & 28 & 64 & & & & & & & 0 & \\
\hline 21 & & & & & 1 & 1 & 0.018 & 9 & 43 & 1 & 1 & 1 & & 1 & & 0.687 & 29 & & & & & & & & & \\
\hline 22 & 1 & 1 & 1 & & & & 0.422 & 24 & 44 & 1 & 1 & 1 & & & 1 & 0.612 & 28 & & & & & & & & & \\
\hline
\end{tabular}

1-FR (+); $\square-F R(-) ; P$ - probability of fatal outcome

\section{Table 5.}

The assessment of the risk of death for the coming year, based on the summation of conventional scores

\begin{tabular}{|c|l|c|c|}
\hline $\mathrm{N}$ & \multicolumn{1}{|c|}{ RF } & Yes & No \\
\hline 1 & NYHA FC $\geq$ III & 15 & 0 \\
\hline 2 & Age $<40$ years & 5 & 0 \\
\hline 3 & SBP $<100$ mmHg & 4 & 0 \\
\hline 4 & $\begin{array}{l}\text { Blockade of the left/right leg } \\
\text { of the bundle of His and/or the } \\
\text { presence of pseudo-infarct Q } \\
\text { wave in ECG }\end{array}$ & 4 & 0 \\
\hline 5 & High-grade VAs & 4 & 0 \\
\hline 6 & LVESV/LVEDV $>0.66$ & 5 & 0 \\
\hline & Score & & \\
\hline
\end{tabular}

$\leq 15$ scores-a low risk of death; 16-22 scores-an average risk of death; 23-28 scores - a high risk of death; > 28 scores - a very high risk of death.

The data in the literature testify to the unfavorable prognostic role of focal myocardial fibrosis in the development of life-threatening episodes of VA in patients with systolic dysfunction of the myocardium. ${ }^{(15,16)}$ A similar association between focal myocardial fibrosis and VAs was demonstrated in our study. In DCM patients, pronounced systolic dysfunction with a pseudo-infarct Q wave in ECG was associated with the presence of high-grade VAs.

In recent years, attempts have been made to create simple algorithms for predicting survival in CHF based on a small number of indicators available in everyday practice. In particular, The Seattle Heart Failure Model (SHFM) is a wellvalidated prediction model of all-cause mortality in patients with HF. SHFM ${ }^{(17)}$ provides an accurate estimate of 1-, 2-, and 3-year survival with the use of easily obtained variables, including clinical characteristics (age, sex, NYHA functional class, systolic blood pressure, and weight), medications (angiotensin-converting enzyme inhibitor, angiotensin receptor blocker, beta-blocker, statin, aldosterone blocker, loop diuretic dose, and allopurinol), device therapies (implantable cardioverter-defibrillator, cardiac resynchronization therapy), and results of diagnostic testing (ejection fraction, lymphocyte percentage, and levels of sodium, hemoglobin, uric acid, and total cholesterol).

M. Kyuma et al. ${ }^{(18)}$ determined the relative risk of death, taking into account the concentration of brain natriuretic peptide, the level of cardiac sympathetic nerve innervations and these indicators simultaneously.

The results of our study are consistent with the data above. In order to identify the most significant predictors of mortality in DCM patients, we have constructed a prognostic model for 
a comprehensive assessment of the risk of death. This method allows eliminating highly correlated RFs and levelling out their complementary impact on a comprehensive assessment of the risk of death. Thus, independent RFs for death in DCM patients are the family form of this pathology, a relatively young age, and NYHA class III/IV, all of which increase the risk of death by 6-10 times. Low BP, abnormal QRS complex, high-grade VAs, low LFEF and increased LVESV (>178 ml) increase the mortality by 1.5-2.5 times. In accordance with the results of MRA, among the clinico-functional parameters, NYHA class III/IV, low BP, a relatively young age, abnormal QRS complex, high-grade VAs and an increase in LVESV/ LVEDV ratio $(>0.66)$ are the most influencing factors for a fatal outcome within 1 year of observation.

\section{Competing interests} interests.

The authors declare that they have no competing

\section{References}

1. Fomin IV, Ageev FT, Arutyunov GP, Bekenkov YuN. [Epidemiology of chronic heart failure in the European part of the Russian Federation]. Russian Heart Failure Journal. 2010; 4:32-42. [Article in Russian].

2. Cleland JG1, Swedberg K, Follath F, Komajda M, CohenSolal A, Aguilar JC, et al.; Study Group on Diagnosis of the Working Group on Heart Failure of the European Society of Cardiology. The EuroHeart Failure survey programme- a survey on the quality of care among patients with heart failure in Europe. Part 1: patient characteristics and diagnosis. Eur Heart J. 2003;24(5):442-63.

3. Belenkov YuN, Mareyev VYu, Ageyev FT. Chronic heart failure. Selected lectures on cardiology. M: GEOTAR-Media; 2006. [In Russian].

4. Reddan DN, Szczech LA, Tuttle RH, Shaw LK, Jones RH, Schwab SJ, et al. Chronic kidney disease, mortality, and treatment strategies among patients with clinically significant coronary artery disease. J Am Soc Nephrol. 2003;14(9):2373-80. 5. Vayhanskaya TG, Kurushko TV, Sivitskaya LN, Danilenko NG, Shestakova LG, Frolov AV. [Algorithm for diagnosis and treatment of patients with dilated cardiomyopathy]. Russian Heart Failure Journal. 2015;6:344-59. [Article in Russian]. 6. Rizun LI, Voronina TS, Dombrovskaia AV, Frolova IuV, Blagova OV, Van EIu, et al. [Noncoronary dilated cardiomyopathy after reverse remodeling heart surgery].
Kardiologiia. 2013;53(12):41-6. [Article in Russian]. 7. Richardson $\mathrm{P}$, McKenna W, Bristow $\mathrm{M}$, Maisch B, Mautner B, O'Connell J, et al. Report of the 1995 World Health Organization/International Society and Federation of Cardiology Task Force on the Definition and Classification of cardiomyopathies. Circulation. 1996;93(5):841-2.

8. Guyatt GH, Sullivan MJ, Thompson PJ, Fallen EL, Pugsley SO, Taylor DW, Berman LB. The 6-minute walk: a new measure of exercise capacity in patients with chronic heart failure. Can Med Assoc J. 1985;132(8):919-23.

9. Shlyakhto EV, Sitnikova MYu. [Modern methods of assessing the prognosis for heart failure]. Russian Heart Failure Journal. 2009;6 (56):322-34. [Article in Russian].

10. Janashia PH, Kruglov VA, Nazarenko VA, Nikolenko SA. Cardiomyopathy and myocarditis: Textbook. M., 2000. [In Russian].

11. Grzybowski J, Bilińska ZT, Ruzyłło W, Kupść W, Michalak E, Szcześniewska D, et al. Determinants of prognosis in nonischemic dilated cardiomyopathy. J Card Fail. 1996;2(2):77-85.

12. Rihal CS, Nishimura RA, Hatle LK, Bailey KR, Tajik AJ. Systolic and diastolic dysfunction in patients with clinical diagnosis of dilated cardiomyopathy. Relation to symptoms and prognosis. Circulation. 1994;90(6):2772-9.

13. Witte K, Thackray S, Banerjee T, Clark AL, Cleland JG. Update of ELITE-II, BEST, CHAMP, and IMPRESS clinical trials in heart failure. Eur J Heart Fail. 2000;2(1):107-12.

14. Schannwell CM, Schoebel FC, Marx R, Plehn G, Leschke $\mathrm{M}$, Strauer BE. [Prognostic relevance of left ventricular diastolic function parameters in dilated cardiomyopathy]. Z Kardiol. 2001;90(4):269-79. [Article in German].

15. Yokokawa M, Tada H, Koyama K, Ino T, Hiramatsu S, Kaseno K, et al. The characteristics and distribution of the scar tissue predict ventricular tachycardia in patients with advanced heart failure. Pacing Clin Electrophysiol. 2009;32(3):314-22. doi: 10.1111/j.1540-8159.2008.02238.x.

16. Dawson DK, Hawlisch K, Prescott G, Roussin I, Di Pietro E, Deac M, et al. Prognostic role of CMR in patients presenting with ventricular arrhythmias. JACC Cardiovasc Imaging. 2013;6(3):335-44. doi: 10.1016/j.jcmg.2012.09.012. 17. Levy WC, Mozaffarian D, Linker DT, Sutradhar SC, Anker SD, Cropp AB, et al. The Seattle Heart Failure Model: prediction of survival in heart failure.Circulation. 2006;113(11):1424-33.

18. Kyuma M, Nakata T, Hashimoto A, Nagao K, Sasao H, Takahashi T, et al. Incremental prognostic implications of brain natriuretic peptide, cardiac sympathetic nerve innervation, and noncardiac disorders in patients with heart failure. J Nucl Med. 2004;45(2):155-63. 